\title{
Análisis de la cadena de valor de la soja en el Paraguay
}

\section{Chain value analysis of soybean and its manufactures in Paraguay}

DOI: $10.46932 / \mathrm{sfjdv2n5-079}$

Received in: Oct 1st, 2021

Accepted in: Dec 30th, 2021

\author{
Rubén Invernizzi Rodas \\ 1Facultad de Ciencias Agrarias - Universidad Nacional de Asunción \\ Ruta Mcal. Estigarribia Km 10,5 \\ San Lorenzo-Paraguay \\ E-mail: rubeninvernizzi@ hotmail.com \\ Victor Enciso Cano \\ 1Facultad de Ciencias Agrarias - Universidad Nacional de Asunción \\ Ruta Mcal. Estigarribia Km 10,5 \\ San Lorenzo-Paraguay \\ E-mail: venciso@agr.una.py \\ Mario León Frutos \\ 1Facultad de Ciencias Agrarias - Universidad Nacional de Asunción \\ Ruta Mcal. Estigarribia Km 10,5 \\ San Lorenzo-Paraguay \\ E-mail: mleonfrutos@yahoo.com
}

\section{RESUMEN}

La producción agrícola conjuntamente con las manufacturas de origen agrícola representa cerca del $32 \%$ del PIB de Paraguay, generan un tercio del empleo y el 77,7\% de los ingresos de divisas para el país. Dentro de los productos agrícolas, la soja y sus derivados representan el $37 \%$ de esos ingresos Aunque el enfoque de la cadena de valor se remonta a varias décadas, la investigación sobre la cadena de valor es reciente en Paraguay. Las existentes no calculan el aporte de cada eslabón. El presente trabajo, a través del enfoque metodológico de Value Links, analiza la cadena de la soja en Paraguay, describiendo y cuantificando la contribución de cada eslabón en valor monetario y relativo. El período de análisis fue el año agrícola 2017/2018 y la información se obtuvo de fuentes secundarias (publicaciones oficiales y páginas web de los gremios, entre otros). Además, recurrió a entrevistas con informantes calificadas como fuente primaria, quienes solicitaron el anonimato. Los resultados mostraron que la producción fue el de mayor contribución al valor agregado (34\%) y la de menor fue el de industrialización (8\%).

Palabras claves: Glycine max, cadena de valor, value links, Paraguay.

\begin{abstract}
Agricultural production together with manufactures of agricultural origin products accounts for $32 \%$ of Paraguay's GDP. It creates one third of employment and $77.7 \%$ of foreign currency income. Soybean and its manufactures, oil and meal, represents $37 \%$ of that income. Although value chain approach goes back several decades ago, research on the subject is quite new in Paraguay. The current literature did not compute nominal nor relative weight of each link. The present paper by means of "value links" methodological approach analyzed soybean chain in Paraguay, describing and quantifying the
\end{abstract}


contribution of each link in monetary and relative terms. The period of analysis was the 2017/2018 marketing year. Secondary information was obtained from official publications and web pages. In addition, interviews were conducted with qualified informants as a primary source, whom requested anonymity. The results had shown that the greatest added value was in the production link (34\%) and lowest one was in the industrialization link $(8 \%)$.

Keywords: Glycine max, Value chain, value links, Paraguay.

\section{INTRODUCCIÓN}

La producción agrícola conjuntamente con las manufacturas de origen agropecuario representa en promedio el 32\% del PIB del Paraguay, genera un tercio del empleo y el 77,7\% del ingreso de divisas al país. Además de su aporte al PIB, la importancia del sector agrícola radica en el hecho de que representa cerca del 90\% de los ingresos por exportación. Las principales exportaciones de Paraguay son bienes primarios (soja, maíz, trigo y arroz). Entre las manufacturas sobresalen básicamente transformaciones iniciales aceite de soja desgomado, harina de soja y carne bovina. La elevada dependencia de la economía paraguaya en la producción y exportación de bienes agrícolas que a su vez son muy dependientes de las condiciones climáticas y de la variación de los precios internacionales incrementa considerablemente la volatilidad del PIB y del desempeño económico del país.

Si bien el planteamiento de cadena de valor en el sentido de encadenamientos, enlaces o eslabones se remonta varias décadas atrás las investigaciones sobre cadena de valor son recientes en Paraguay. Las existentes mayormente descripciones de los actores pero carecen de estimaciones cuantitativas del aporte de cada eslabón, sino que más bien de cuantifican la cadena en su conjunto. El presente trabajo mediante el enfoque metodológico Value links desarrollado por la Agencia de la Cooperación Técnica Alemana (GIZ), supera esta limitación y cuantifica el aporte o valor bruto agregado de cada componente.

Usando la citada metodología, el objetivo de esta investigación fue caracterizar la cadena de valor de los productos derivados de la industrialización de soja de Paraguay. Específicamente se apunta a identificar cuáles son los eslabones de la cadena, lo que igualmente se conoce como mapeo; describir cada uno de los eslabones de la cadena; y finalmente analizar económicamente la cadena.

\section{ANTECEDENTES}

\subsection{IMPORTANCIA DE LA CADENA DE LA SOJA EN PARAGUAY}

Hasta la década del 60 el Paraguay era una sociedad agraria, basada en una agricultura atrasada y estancada (Campo, 2010). En la década del setenta, la economía del Paraguay vivió años de excepcional crecimiento relacionados con la construcción, conjunta con Brasil, de la represa de Itaipu. Desde principios de los ochenta el Paraguay entró en una senda de bajo o nulo crecimiento económico y no fue 
capaz, de mantener un nivel de crecimiento superior a la de la tasa poblacional sino a partir del 2008 (Campo, 2010), año en que los precios de los commodities agroalimentarios experimentaron un alza histórica (Fondo Monetario Internacional [FMI], 2018). Entre el 2008 y el 2017, el PIB de Paraguay llegó en un par de ocasiones a crecer a tasas de dos dígitos inclusive (FMI, 2018).

Si bien el sector terciario (servicios) es el principal componente del PIB paraguayo seguido del secundario, quedando en tercer lugar el sector primario, liderado por la agricultura, el desempeño económico del Paraguay está fuertemente ligado a la producción y exportación agrícola, en especial de los principales productos de exportación (soja, carne vacuna y maíz) (Oficina Económica y Comercial de España en Asunción [OECE], 2017). Entre el 2015 y el 2018, las exportaciones paraguayas, excluidas energía eléctrica, sumaron en promedio 8.500 millones de dólares. La soja y sus derivados representaron el 37 \% (BANCO CENTRAL DEL PARAGUAY, 2018).

La producción agrícola conjuntamente con las manufacturas de origen agropecuario representa el $32 \%$ del PIB, genera un tercio del empleo y el 77,7\% del ingreso de divisas al país (BCP, 2015; BCP, 2018). Además de su aporte al PIB, la importancia del sector agrícola radica en el hecho de que representa cerca del 90\% de los ingresos por exportación (BCP, 2015; BCP, 2018). La elevada dependencia de la economía paraguaya en la producción y exportación de bienes agrícolas que a su vez son muy dependientes de las condiciones climáticas y de la variación de los precios internacionales incrementa considerablemente la volatilidad del PIB y del desempeño económico del país. Según el Banco Mundial (BANCO MUNDIAL, 2014) "las crisis relacionadas con el clima representan por sí solas más del 50\% de la volatilidad del PIB agrícola”.

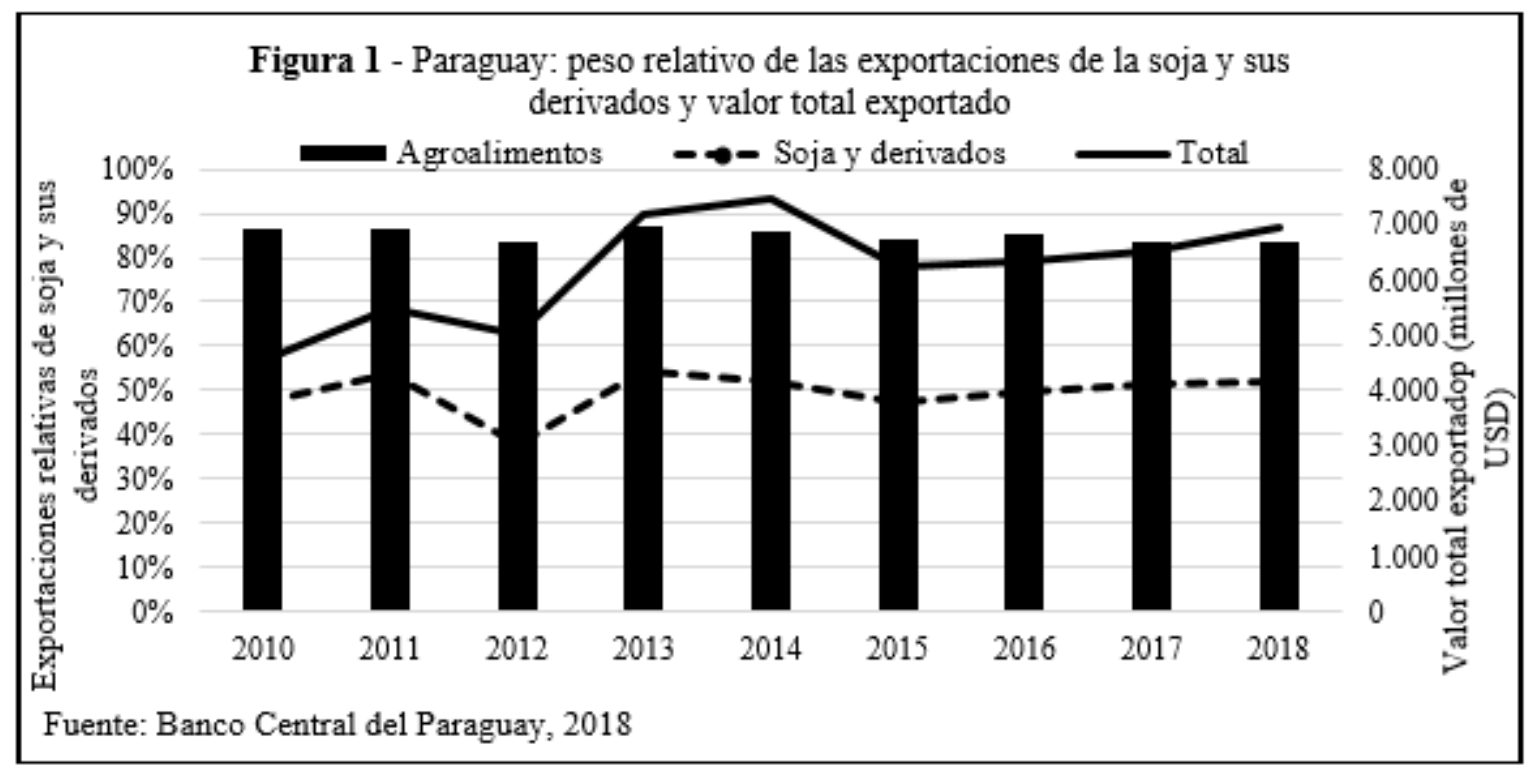




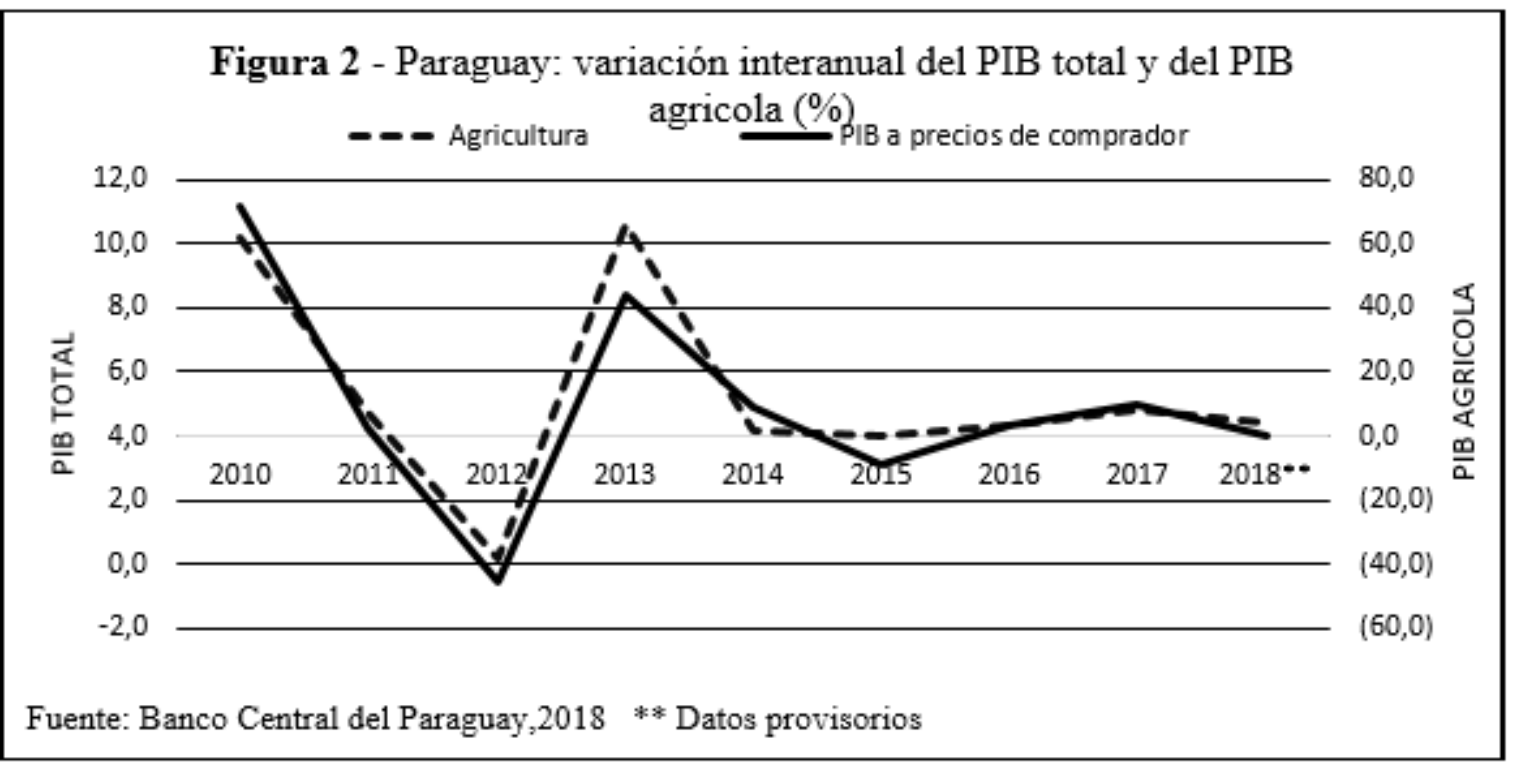

\subsection{CONCEPTO DE CADENA DE VALOR}

Si bien el planteamiento de cadena de valor en el sentido de encadenamientos, enlaces o eslabones se remonta Albert Hirschman en 1958 (Chávez, 2012), el desarrollo teórico de cadena de valor fue introducido por Michael Porter a mediados de los ochentas en su seminal publicación Ventaja Competitiva en 1985 (Porter, 2006). Tal publicación puede ser considerada como el primer aporte teórico al concepto, (Heredia y Huarachi, 2009). El principal aporte de Porter fue la introducción del concepto de sistema de valor como un todo compuesto de cadenas de valor de los proveedores, de la empresa, de los canales y de los clientes (Chávez, 2012). Estas actividades, Porter las dividió en actividades primarias o en línea, que son las directamente asociadas a la obtención del bien e incluye la logística de entrada, las operaciones transformadoras, la logística de salida, mercadotecnia y ventas, y servicios. Entre las actividades secundarias, a las que identificó como las que agregan valor al bien en forma indirecta cumpliendo más bien un rol de ayuda a las actividades primarias. Se incluye la infraestructura de la empresa, la gestión de los recursos humanos, el desarrollo de la tecnología, y el aprovisionamiento, (PORTER, 2006).

El concepto de cadena de valor se suele confundir o usar como sinónimo de cadena productiva. FAO (2015) señala que una cadena de valor es una red o alianza estratégica entre participantes de una cadena productiva con el objeto de acordar estrategias de producción diferenciadas o de cooperación, en tanto que la cadena productiva se refiere a las interacciones entre los actores del mercado desde el inicio al final de la cadena. Pero, ¿cuál es la utilidad de la cadena de valor? Se cuestiona y a la vez responde Chávez (2012), señalando que la utilidad de caracterizar una cadena de valor es que provee las bases para avanzar hacia el análisis de cadena de valor. Este análisis permite saber si se está aprovechando las capacidades y los recursos de las empresas, las especificidades de los actores; igualmente es una ayuda para identificar las actividades que mayores beneficios aportan, así como lo opuesto. A decir de FAO 
(2015) ubicar fuentes de ventaja potencial entre las actividades y procesos de la cadena y de sus participantes.

La agencia de cooperación alemana basada en el concepto de cadena de valor desarrolló la metodología conocida como "value links". La concibe como una compilación sistemática de métodos orientados a la acción para promover el desarrollo económico de los actores (Springer-Heinze, 2008). Debe sin embargo quedar en claro, que value links no es un nuevo concepto aunque tiene ciertas características peculiares respecto a la conceptualización standard de cadena de valor. Estas particularidades se definen en orientación hacia el mercado, perspectiva de cadena como sistema, liderazgo privado, cambio estructura y formación de capacidad (SPRINGER-HEINZE, 2008).

\subsection{CADENAS DE VALOR EN PARAGUAY}

Existen varias investigaciones sobre cadenas de valor en Paraguay. Setrini (2014) por encargo de JICA coordinó una investigación de cadenas buscando identificar aquellas con potencial de ser implementadas con pequeños productores. Analizó las cadenas de maíz, soja, trigo, caña de azúcar y mandioca. Concluyó que se debe "priorizar el desarrollo de las cadenas de mandioca y caña de azúcar", teniendo en cuenta que "los mercados de exportación para los productos derivados (azúcar orgánica, etanol y almidón) de estos productos demuestran una tendencia de crecimiento y apertura hacia las exportaciones potenciales en Paraguay". Con respecto a los granos concluye que la cadena de granos si bien tiene potencial de crecimiento, al igual que sus manufacturas, para los pequeños productores no es competitivos en la producción de maíz, soja ni trigo.

Ortiz y Riquelme (2016) analizaron específicamente la cadena de las oleaginosas, en un trabajo apoyado por la Cámara Paraguaya de Procesadores de Oleaginosas y Cereales (CAPPRO), y cuantifican las ventajas de reducir las exportaciones de soja en grano y aumentar las de sus derivados (aceite y harina principalmente), como una primera etapa para luego fomentar la transformación, de la harina, en carne. Los autores señalan que cada tonelada de soja significaba en el año del estudio un ingreso de 348\$/t, contra $803 \$ / t$ en promedio por la exportación de harina y aceite, mientras que por carne se pagaba un promedio de 3.185\$/t. El trabajo concluye con una propuesta de plan industrial, uno de cuyos fundamentos cuantitativos establece que si se eleva el procesamiento de la soja al 65\% y toda la harina se transformara en proteína animal, en el 2025, las exportaciones (carnes de todo tipo, lácteos y huevos) serian de unos 3.302 millones de dólares.

Una tercera fuente de investigación sobre cadenas en Paraguay es el proyecto cadenas de valor de USAID, con investigaciones en cadenas de maíz, caña de azúcar, hierbas medicinales, yerba mate y banano. Los trabajos concluyen con propuestas para el desarrollo de dichas cadenas con pequeños agricultores en la zona norte del Paraguay. 
Una de las limitaciones en estos trabajos es la falta de cuantificación del valor bruto y del valor agregado de cada eslabón de la cadena, con alguna salvedad del trabajo de Ortiz y Riquelme (2016). Esta situación es abordada en la presente investigación mediante la aplicación de la metodología de value links, de modo que al final se pueda tener una visión del aporte nominal y relativo de cada eslabón de la cadena. Esto se convierte así en su principal aporte.

\section{OBJETIVO}

El objetivo general de este trabajo es caracterizar la cadena de valor de los productos derivados de la industrialización de soja de Paraguay. Específicamente se apunta a identificar cuáles son los eslabones de la cadena, lo que igualmente se conoce como mapeo; describir cada uno de los eslabones de la cadena; y finalmente analizar económicamente la cadena.

\section{METODOLOGIA}

Esta investigación fue de carácter descriptivo en el que se expusieron datos y análisis de rasgos, cualidades y atributos de la cadena de la soja y en sus manufacturas, a saber aceite y harina. Además, se presentó el mecanismo de intercambio comercial entre los actores en los distintos eslabones de una cadena de valor. Se utilizó el enfoque metodológico Value links desarrollado por la Agencia de la Cooperación Técnica Alemana (GTZ); específicamente se utilizó el Módulo 2 "Análisis de una cadena de valor" (Springer-Heinze, 2008). Dicho módulo establece que el análisis de las cadenas de valor comprende una serie de diferentes métodos, agrupados en tres tareas básicas, que en el presente trabajo se combinaron en dos: (i) mapeo y cuantificación de la cadena de valor, y (ii) análisis económico de la cadena de valor. El mapeo consistió en el trazado de un mapa básico de la cadena de valor, identificando los principales eslabones y visualizando la secuencia de funciones de producción y comercialización, los operadores y los vínculos comerciales entre los mismos. Se agregaron al mapeo y cuantificación, los prestadores de servicios y los actores que apoyan la cadena a nivel macro. El análisis económico de cadenas de valor incluyó la cuantificación del valor agregado total generado por la cadena de valor y la participación relativa en los diferentes eslabones. Esto luego permitió cuantificar el aporte en cada eslabón. Se aplicó la siguiente fórmula en cada eslabón:

\section{Valor agregago $(V A)=$ Valor total del Venta - Valor total de los bienes intermedios}

Las fuentes primarias de información fueron básicamente entrevistas con actores claves de la cadena, que en todos los casos prefirieron mantener el anonimato. Acceso a información primaria, en especial de industrias, sigue siendo complicado en Paraguay. Los datos de fuentes secundarias utilizados fueron: (i) datos de páginas web de organismos gremiales nacionales, a saber Cámara Paraguaya de 
Productores de Cereales y Oleaginosas (CAPECO), de la Cámara Paraguaya de Procesadores de Oleaginosas (CAPPRO), Cámara Paraguaya de Automotores y Maquinarias (CADAM), e internacionales tales como FAO y USDA; (ii) literatura sobre el tema, extraídas de materiales bibliográficos de la Facultad de Ciencias Agrarias, de Organismos Internacionales, de informes gubernamentales con datos oficiales del Ministerio de Agricultura y Ganadería (MAG), y del Banco Central del Paraguay (BCP). El periodo de análisis fue el año agrícola 2017/2018.

\section{DESARROLLO}

\subsection{MAPEO DE LA CADENA DE VALOR DE LA SOJA Y SUS MANUFACTURAS}

El mapeo muestra que la cadena de valor cuenta con cinco eslabones principales, a saber (i) provisión de insumos específicos, (ii) producción de materia prima, (iii) acopio, (iv) industrialización, y (v) ventas.

\subsubsection{Provisión de insumos}

Consiste en la provisión insumos agrícolas a los productores. Los insumos se agrupan en semillas e insumos técnicos, que incluye insecticidas, fungicidas y fertilizantes principalmente. El nivel de profundidad de las empresas que operan en el eslabón es variado. Algunas empresas se especializan en proveer un solo insumo, por ejemplo semillas o tanto semillas como insumo técnico, o solamente insumos técnicos. Sin embargo la mayoría son diversificadas y proveen al productor tanto de insumos técnicos como del material genético.

Los proveedores de semillas son en total 51 empresas, muchas de las cuales además importan y comercializan agroquímicos y fertilizantes. Gremialmente se agrupan en la Asociación de Productores de Semillas del Paraguay (APROSEMP) (APROSEMP, 2017a). Existe igualmente existe una cámara que agrupa tanto quienes comercializan semillas como defensivos, Cámara de Fitosanitarios y Fertilizantes, (CAFyF), en tanto que las industrias se agrupan en la Cámara Industrias Nacionales de Defensivos Agrícolas (CINDA) (CINDA, 2019).

Las semillas comercializadas pertenecen a los siguientes obtentores Don Mario S.A., COODETEC, Criadero Santa Rosa Ltda./Granar S.A., EMBRAPA, Ing. Agr. Luis Alberto Curti, Ing. Agr. Francisco Terasawa, Monsanto Argentina S.A.I.C., Nidera S.A., Relmo S.A. y Syngenta Seeds Ltda. Las empresas que proveen semillas e insumo más reconocidos por su antigüedad en el mercado son: Agro Santa Rosa S.A.E., Compañía Dekalpar S.A., GPSA, Agro Silo Santa Catalina, Sem Agro S.A.E., Cooperativa Yguazú Ltda. Cooperativa Pindó Ltda., y Cooperativa Colonias Unidas (APROSEMP, 2017b). 
Entre las marcas comerciales de los defensivos agrícolas existen en el mercado productos tanto de multinacionales (Bayer, Basf, Dupont), como de empresas locales que importan los componentes y lo mezclan localmente (Tecnomyl, Diagro, Dekalpar y Chemtec entre las más reconocidas). Existen otras empresas que importan y comercializan productos genéricos desde China e India. El valor de estos insumos y la semilla se estima totalizó 1.800 millones de dólares en el 2018. Igualmente forman parte del eslabón las empresas proveedoras de maquinarias e implementos agrícolas.

En fertilizantes, las marcas comerciales utilizadas y representadas en el Paraguay son Fertipar, Isusa, Mosaic, Buena Zafra, ECOP, Bunge entre otros. Una buena parte de los fertilizantes comercializados localmente son formulados y mezclados en el país. En los últimos nueve años (20102018) las importaciones registradas de fertilizantes han promediado 400.000.000 de dólares (BCP, 2018). En este periodo los fertilizantes con dos o tres elementos (NPK) han reducido su participación desde el $83 \%$ al $60 \%$, ante las inversiones en facilidades para formular localmente de acuerdo a las necesidades específicas de los productores. Brasil es el principal proveedor del mercado paraguayo con una cuota cercana al 60\%; otros proveedores son Rusia, Bielorrusia y China. En el 2010, las importaciones registradas de insecticidas, fungicidas, herbicidas, inhibidores de germinación y reguladores de crecimiento sumaron 9,93 millones de dólares, valor que creció 600\% para el 2018 cuando totalizó 69,59 millones de dólares. Las importaciones de insecticidas representaron el 53\% del valor, quedando el saldo en partes iguales entre los herbicidas, inhibidores y reguladores por un lado y los fungicidas por el otro (BCP, 2018).

Las empresas proveedoras de maquinarias, agrupadas en la Cámara Distribuidora de Automotores y Maquinarias (CADAM), están especializadas en esta línea, y no se involucran en el negocio de insumos ni semillas. Estas empresas prestan lo que se puede denominar servicios operacionales. Las importaciones de tractores, cosechadoras y pulverizadoras, luego de caer al mínimo en el 2016, se han recuperado en el 2017 y 2018, aunque sin llegar a los valores del 2011, que según CADAM, s/d fue el mejor año en la presente década. A efectos de dimensionar, las importaciones de tractores, pulverizadoras y cosechadoras sumó en el 20114054 unidades, bajo a 1239 en el 2016 y se recuperó a 2725 en el 2018. En términos de valor monetario las importaciones de cosechadoras por ejemplo han representado en el 2011 122.000.000 de dólares, cayendo a 22.500.000 en el 2016 y ha repuntado a 80.300.000 en el 2018 (BCP, 2018).

\subsubsection{Producción de materia prima}

Está conformada básicamente por productores a los que se los puede clasificar en dos grupos. Por un lado, están los productores organizados en cooperativas de producción quienes obtienen insumos de sus cooperativas, a las cuales igualmente entregan su producción cerrando el círculo. La cooperativa luego se encarga de negociar el mejor precio, y en algunos casos hasta exportar o procesarlas. Por el otro lado 
están los demás productores que obtienen insumos de empresas privadas y que por lo general entregan su producción al silo para su almacenamiento; venden una parte suficiente para honrar sus créditos, negociando el resto buscando la mejor cotización. Hasta cierto grado se puede afirmar que tienen mayor flexibilidad a la hora de adquirir los insumos al igual que mayor libertad para comercializar su producción.

En lo gremial, los productores se asocian en diversas organizaciones, que se aglutinan en la Unión de Gremios de la Producción (UGP). Algunas de las organizaciones son la Cámara Paraguaya de Exportadores y Comercializadores de Cereales y Oleaginosas (CAPECO), la Coordinadora Agrícola del Paraguay (CAP), la Federación de Cooperativas de Producción (FECOPROD), y la Asociación de Productores de soja, Cereales y Oleaginosas del Paraguay (APS).

La producción de soja para grano comercial (para diferenciarla de las semillas), ha experimentado un crecimiento constante, prácticamente lineal, con algunos años de reducciones que en ciertos casos fueron muy importantes. Datos de la página web de CAPECO (CAPECO, s/da) muestran que en la zafra 1996/1997 se cosecharon 2.771 millones toneladas de soja; diez años más tarde se pasó la marca de 5.000 millones. En el 2016/2017, se marcó record para el país y se cosecharon 10.336 millones de toneladas. En el mismo periodo, la superficie se amplió desde 1.050.00 hectáreas a 3.400.000 hectáreas en la zafra 2017/2018. Por su lado el rendimiento ha estado en torno a los $2.500 \mathrm{~kg} / \mathrm{ha}$ hasta el 2015/2016. En las dos últimas zafras ha sobrepasado los $3.000 \mathrm{~kg}$. Datos oficiales, (Ministerio de Agricultura y Ganadería [MAG], 2008) reportan que la superficie sembrada en la zafra 2017/2018 fue de 3.510.000 hectáreas, experimentado un aumento de 3,8 \% con relación a la zafra 2016/17. La producción totalizó 11.045.971 toneladas, que comparando con la zafra anterior se produjo un aumento del 5,4\%. El rendimiento promedio fue de $3.147 \mathrm{~kg} / \mathrm{ha}$., en tanto que en la zafra previa fue de $3.100 \mathrm{~kg} / \mathrm{ha}$.

El 78\% de la superficie de siembra de la oleaginosa se concentra en 4 Departamentos: Alto Paraná (27\%), Canindeyú (19\%), Itapúa (18\%) y Caaguazú (13\%). En lo referente a la producción estos Departamentos son los principales, con la particularidad que Itapúa produce más soja que Canindeyú, debido a una mayor productividad. Es así que el primero la media estuvo en $3.458 \mathrm{~kg} / \mathrm{ha}$, mientras que en el segundo el rendimiento medio estuvo levemente por debajo de los $3.000 \mathrm{~kg}$. Amambay presentó el mayor rendimiento alcanzado $3.484 \mathrm{~kg} / \mathrm{ha}$. En la Región Occidental se sembraron 5.315 hectáreas que con una productividad media de $1.987 \mathrm{~kg} / \mathrm{ha}$ permitió la cosecha de 10.562 toneladas. El 90\% de la producción se encuentra en el Departamento de Boquerón donde la soja rindió más de 2.000 kg/ha (MAG, 2018; CAPECO s/da)

La producción nacional de semilla ha pasado de 780.583 bolsas de $40 \mathrm{~kg}$ en 1997 a 1.316 .370 bolsas en 2013 (últimos datos obtenidos de la página web de APROSEMP). Tal crecimiento ha sido del $69 \%$, en tanto que la importación de semilla se incrementó en un76\%. Por su lado la demanda de la simiente creció en $200 \%$ en el mismo periodo, con lo cual el uso de semillas ofertadas por productores 
semilleristas e importadores se redujo desde un 67\% en 1997 a tan solo 38\% en el 2013 (APROSEMP, 2017b). La diferencia de cubierta con semilla no registrada, y con semillas propias del productor.

\subsubsection{Acopio}

Consiste en recibir el grano de soja y acondicionarlos para su conservación y comercialización. Los acopios o silos pueden pertenecer a las industrias o acopiadores "no industriales" (incluyendo cooperativas), que luego negocian con las industrias o con las exportadoras. Las grandes empresas productoras y las cooperativas poseen sus propios silos y generalmente negocian directamente con la industria o con la exportación, debido al gran volumen que manejan lo que les da ventajas. Las empresas que cuentan con silos adquieren la producción de soja, y realizan un descuento al productor denominado "Premio". Este es un descuento sobre la cotización del contrato Mayo en la Bolsa de Chicago. Tal descuento se destina a cubrir los costos logísticos para transportar el producto hasta los puertos y para cubrir los costos operativos de los silos. En promedio el valor del premio estuvo alrededor de 50 USD/t en la zafra 2017/2018.

En Paraguay existen aproximadamente 700 acopios distribuidos en nueve departamentos. Más del 90\% de estos silos son destinados a los granos de soja y maíz. Cada acopio tiene en promedio una capacidad de almacenamiento de 17.000 toneladas. En total la capacidad estática de estos acopios es de 11,5 millones de toneladas en el año 2018, que comparativamente con el último dato previo (2015) significa un aumento de 6.50\%. Los Departamentos con mayor estática en el 2018 son igualmente los de mayor producción, Alto Parana (31\%), Itapua (19\%), Canindeyu (15\%) y Caaguazu (10\%) (CAPECO, s/db). La capacidad del Departamento Central, es levemente superior a la de Caaguazu se debe a que plantas industriales importantes como CAISA, ADM, y Conti Paraguay además de otras agroexportadoras importantes tienen sus centros de acopio en el Departamento Central al margen del Rio Paraguay.

Los operadores de mayor capacidad estática en Paraguay son en su mayoría empresas que cuentan con industrias procesadoras de soja. Entre los diez operadores de silos con mayor capacidad estática se encuentran Cargill, ADM, Cooperativa Colonias Unidas, y CAIASA que tienen el $21 \%$ de la capacidad de almacenamiento total. Sin embargo cerca de dos tercios de la capacidad de acopio se encuentran dispersa entre un gran número de acopios y empresas que tienen el poder de negociar lo recolectado con las industrias o exportarlos directamente. 


\subsubsection{Industrialización}

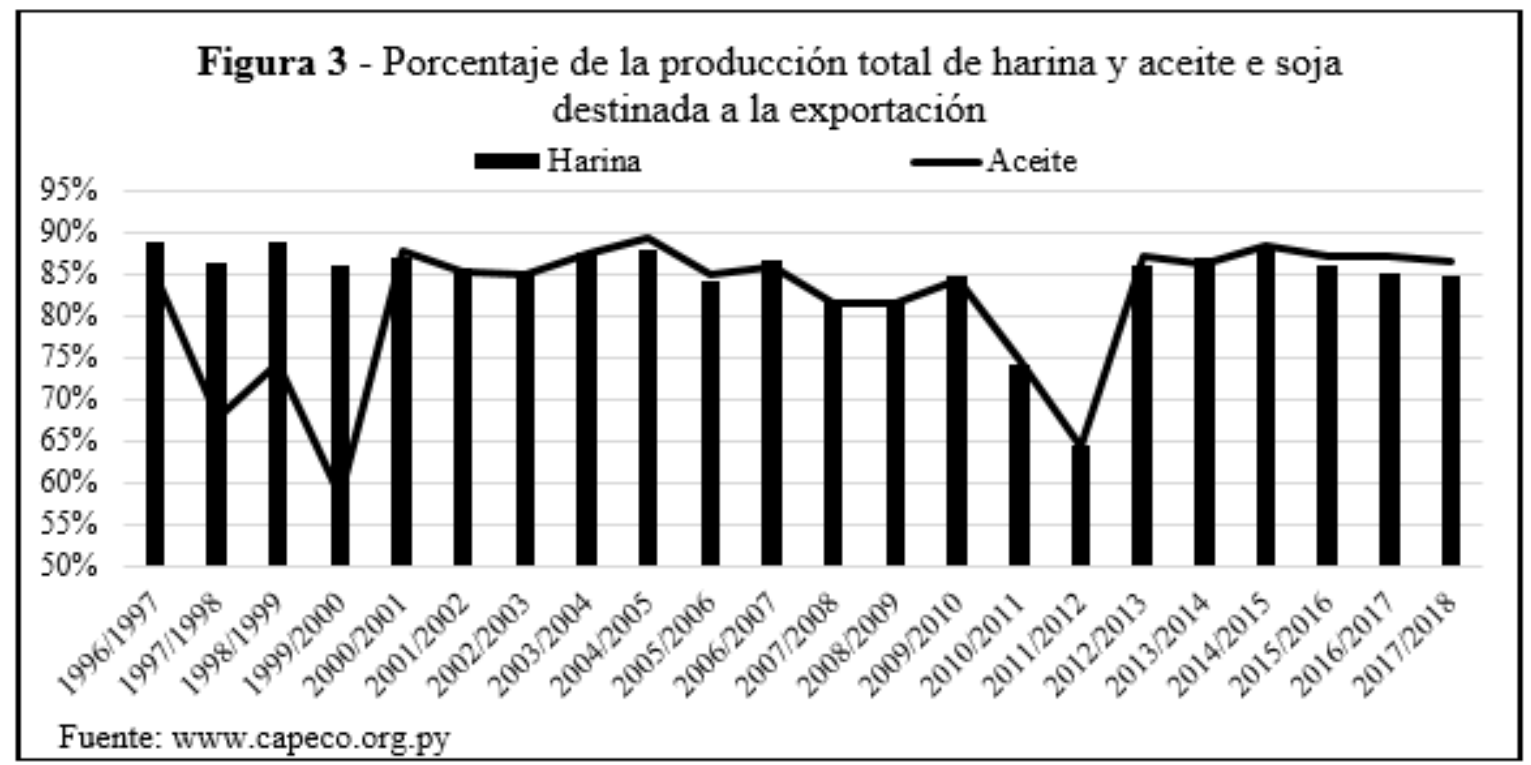

Consiste en la conversión de la materia prima en tres productos básicos, que son el aceite de soja crudo desgomado, la harina de soja y la cascarilla de soja. Paraguay cuenta con siete plantas industriales principales, con una capacidad instalada de molienda de aproximadamente 14.300 toneladas de soja al día (Ortiz y Riquelme, 2016). Se estima que estas industrias emplean aproximadamente a 5.600 personas en forma directa e indirecta (Ortiz y Riquelme, 2016). Según datos de la CAPPRO (2013a), la inversión en este sector de los miembros de esta Cámara es de 1.100 millones de dólares. Hasta el 2013, la capacidad de molienda del sector fue de 2.800.000 toneladas y un uso cercano al 96\%. Desde el 2014 entran en operación CAISA y la de ADM, llevando la capacidad instalada a 5.000.000 de toneladas, un poco más del $50 \%$ de la producción nacional del grano de soja. La capacidad utilizada en el primer año de la ampliación fue de $63 \%$, subiendo diez puntos porcentuales al siguiente año, estancándose en ese nivel desde entonces. CAPECO (s/dc) presenta datos para un periodo de tiempo mayor que permite apreciar la evolución del uso de la soja, especialmente entre exportación y procesamiento, y que se presenta en el gráfico CAPECO.

Las principales empresas, medidas por su capacidad de procesamiento, asociadas a la Cámara son: CAIASA (Complejo Agro Industrial Angostura S.A.), un joint venture, entre Compañía Paraguaya de Granos S.A., Bunge Paraguay S.A. y LDC Paraguay S.A. Las otras grandes son las multinacionales Cargill y ADM ubicadas en Minga Guazú (Alto Paraná) y Villeta (Central) respectivamente. completan el mapa de la cadena de valor son la de MERCO S.A. de LDC Paraguay ubicada en el Departamento de Caaguazú, Conti Paraguay S.A., ubicada en Capiatá, Cooperativa Colonias Unidas en Itapúa al igual que la planta de Oleaginosa RAATZ S.A. Las empresas procesadoras se encuentran asociadas en la Cámara Paraguaya de Procesadores de Oleaginosas y Cereales (CAPPRO). 


\subsubsection{Mercado}

La producción de soja paraguaya tiene como destino principal el mercado externo. La exportación de grano de soja se ha mantenido constante alrededor del $70 \%$ hasta la entrada en funcionamiento de las grandes procesadoras en el 2013 (BCP, 2018). Desde entonces, el envío de granos en estado natural ha estado cerca del 60\%. La Unión Europea es el tradicional mercado de granos de soja de Paraguay. En los últimos años sin embargo, la demanda argentina ha sido más agresiva pasando a ocupar el primer lugar. Esto fue producto de la combinación de dos factores; por un lado la fuerte caída de la producción de soja en Argentina en la zafra 2017/2018, y el levantamiento de las restricciones para importar soja paraguaya, a la vez relacionado con las importantes inversiones en la zona de Rosario para la ampliación de la capacidad de molienda.

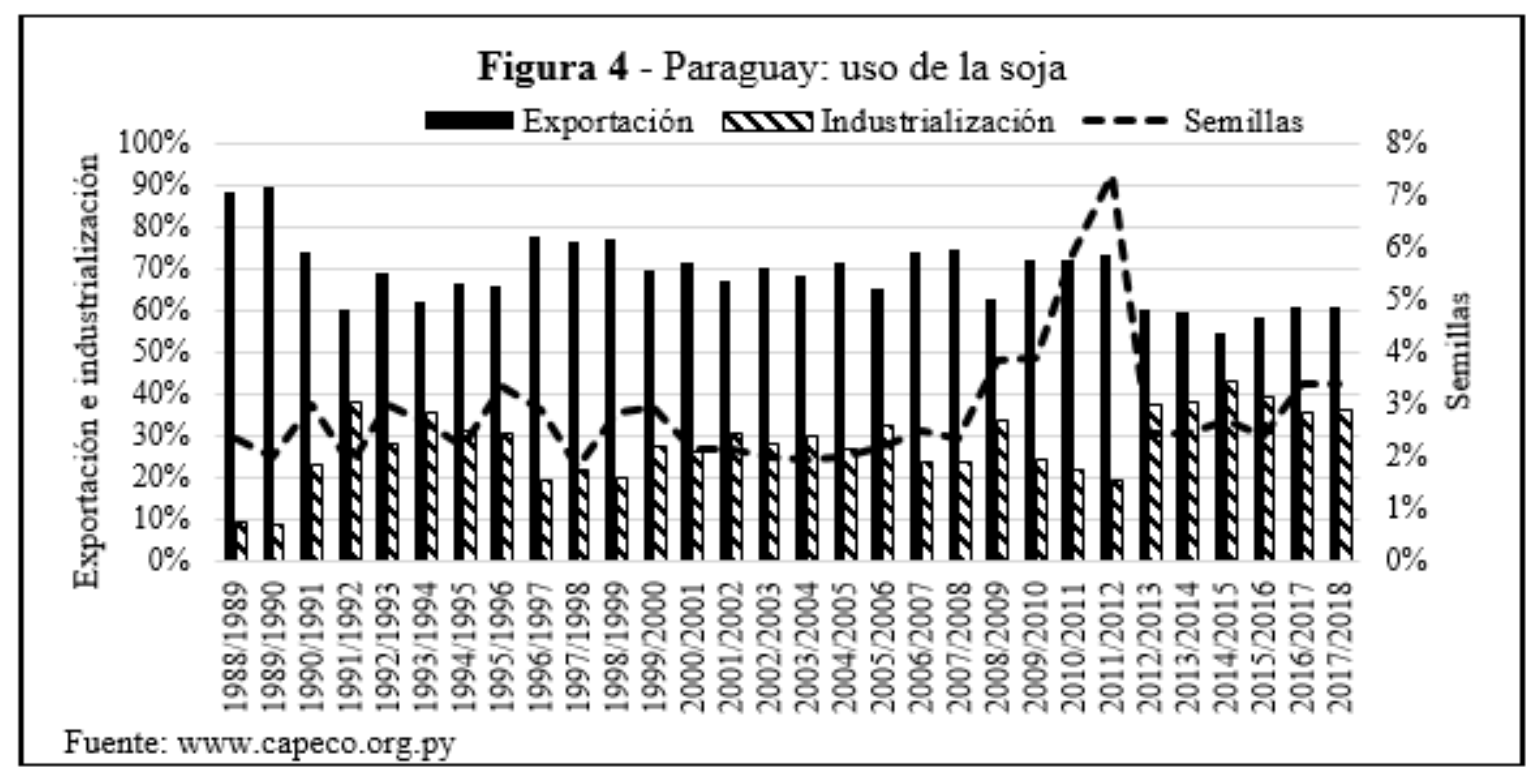

Las industrias exportan la mayor parte de su producción. Una pequeña porción se comercializa en el mercado interno, ésta aún no es significativa para la cadena. Según CAPPRO (2013b) el aceite y la harina de soja en el año 2017 se han exportado en un 92\% y 91\% del total producido respectivamente. Igualmente, las industrias exportan parte del grano acopiado en forma natural sin procesamiento. Los datos de que cubren un mayor periodo de tiempo, sin embargo muestran que la exportación de ambos productos está cerca del 85\%. La diferencia se debe cuestiones metodológicas en el tiempo considerado. CAPECO considera el año comercial (setiembre a agosto) en tanto CAPPRO contabiliza enero a diciembre.

Los principales importadores de aceite de soja de Paraguay han sido históricamente los países asiáticos, con una participación del 64\% entre el 2010 y el 2018, especialmente India y Bangladesh, que entre el 2010 y el 2018 han importado el 50\% de los envíos externos de Paraguay (BCP, 2018). Los países del MERCOSUR, más específicamente Argentina y Brasil han sido destino del 14\% de las exportaciones, 
seguido de la UE con un peso relativo del 8\% en el mismo periodo (BCP, 2018). El principal mercado de la harina de soja paraguaya es la Unión Europea, con una participación media en el mismo periodo antes citado del 40\%. Luego se encuentra Perú con el 32\% (Chile 18\% y Perú 14\%), y Asia con el $12 \%$ (Indonesia 5\% y Japón 3\%) (BCP, 2018).

\subsubsection{Otros actores de la cadena}

Igualmente forman parte de la cadena las entidades prestadoras de servicios. Entre estas se citan a la banca pública y privada que otorgan servicios financieros a los operadores de la cadena. En el mismo sector se encuentran las empresas que brindan servicios de asistencia técnica (tanto agronómica como contable y de gestión), venta de insumos y maquinarias, empresas transportadoras terrestres y fluviales. Por último, encontramos en la misma Figura a las Asociaciones y Cámaras (APROSEMP, CAPECO, CAPPRO) que aglutinan a las empresas que se desempeñan en cada eslabón, apoyándolas por medio de la articulación entre el sector público y privado para obtener mayores beneficios y capacidad para generar mejores resultados en todos los aspectos relacionados al sector.

Los principales prestadores de servicio de apoyo a nivel macro en la cadena son los entes reguladores del buen funcionamiento, y armonía entre todos los actores de los eslabones de la cadena y configura a su vez el entorno a través de leyes que se deben respetar. Las de mayor incidencia se citan a continuación: (i) Servicio Nacional de Calidad y Sanidad Vegetal y de Semillas (SENAVE), que regula la comercialización y utilización de los insumos de uso agrícola en los cultivos, cuida la calidad las semillas, su biotecnología, y protege el derecho de sus creadores, y habilita a los profesionales para el asesoramiento técnico a los operadores de la cadena, desde el eslabón correspondiente a insumos específicos hasta el eslabón de ensilado (SENAVE.GOV, s/d). (ii) Ministerio de Trabajo, Empleo y Seguridad Social (MTESS) aplica y regula la normativa laboral vigente de las relaciones entre los empleadores y los empleados contratados por las empresas para los servicios (MTESS.GOV, s/d). (iii) Instituto Paraguayo de Tecnología Agraria (IPTA) aporta investigación en tecnología agraria para el mejoramiento de la producción, aporta nuevas variedades de semillas y mecanismos de siembra más productivos que pueden apoyar al eslabón de insumos específicos y producción (IPTA.GOV, s/d). (iv) Ministerio de Industria y Comercio (MIC) es el promotor de la actividad de los operadores de la cadena entre sí y coordinador de la inserción de los productos industrializados en el mercado internacional (MIC.GOV, 2014). (v) Aduana es la entidad facilitadora del comercio internacional, responsable de la eficiente recaudación de los tributos y fiscalizador del tráfico de mercaderías, tanto de la importación de insumos para uso de los operadores, como de exportación de productos terminados de la industria (ADUANAS.GOV, 2010). (vi) Subsecretaría de Estado de Tributación (SET) es la institución reguladora 
del cumplimiento de las leyes tributarias vigentes y responsable de la recaudación de los impuestos que los operadores pagan al estado por la actividad comercial que estas realizan (SET.GOV, s/d).

\subsection{ANÁLISIS ECONÓMICO DE LA CADENA DE VALOR DE LA INDUSTRIALIZACIÓN DE LA} SOJA.

\subsubsection{Insumos específicos}

La superficie de siembra de soja en Paraguay en la zafra 2017/2018 fue 3.510.000 hectáreas que en base a un rendimiento de $3.147 \mathrm{Kg} /$ ha dio una producción de 11.045971 toneladas (MAG, 2018). Datos proveídos por el área de economía rural de la Facultad de Ciencias Agrarias de la Universidad Nacional de Asunción1 indican que el costo medio de una hectárea de soja fue de 611 \$/ha, de los cuales 420 \$/t fueron insumos técnicos (semillas, defensivos agrícolas y fertilizantes) y el saldo 193 \$/t correspondió a insumos físicos, igual a horas tractor y horas hombres. Con estos datos se aplicó la fórmula de valor agregado, dando por resultado que el valor del negocio en este eslabón de la cadena es de es de 1.467 millones de dólares. Debido a que es el estadio inicial, se carece de bienes intermedios.

$$
\begin{aligned}
& \text { Valor agregado }(\mathrm{VA})=\text { Valor total de ventas }- \text { bienes intermedios } \\
& \mathrm{VA}=(\text { Sup. sembrada } \mathrm{x} \text { costo de insumos técnicos })-(0) \\
& \mathrm{VA}=3.510 .000 \text { ha } \times 420 \frac{\$}{\mathrm{ha}}=1.467 .180 .000 \$
\end{aligned}
$$

\subsubsection{Producción}

La producción de la zafra, tal como se indicó más arriba fue de 11.045.971 toneladas. La misma fuente que brindó los datos de costos, señaló que el precio medio recibido por los agricultores por cada tonelada de soja en finca fue de 371\$/t, premio incluido, que en la zafra promedió 50\$/t según la misma fuente. A un precio de venta bruto de 371\$/t, el valor de venta de la soja fue de 4.098 millones de dólares. Los bienes intermedios consistieron en el costo de producción, que en el caso de esta investigación fue el costo directo, dio un valor de 2.145 millones de dólares. Con estos datos se aplicó la fórmula de valor agregado, dando como resultado que el valor agregado del eslabón es de 1.953 millones de dólares.

Valor agregado $(\mathrm{VA})=$ Valor total de ventas - bienes intermedios $\mathrm{VA}=($ Producción $\mathrm{x}$ precio de venta bruto $)-($ Superficie $\mathrm{x}$ costos directos $)$

$$
\begin{aligned}
& \mathrm{VA}=\left(11.045 .971 \$ \mathrm{t} \times 371 \frac{\$}{\mathrm{t}}\right)-\left(3.510 .000 \text { ha } \mathrm{x} 611 \frac{\$}{\mathrm{ha}}\right) \\
& \mathrm{VA}=4.098 .055 .241 \$-2.144 .610 .000 \$ \\
& \mathrm{VA}=1.953 .445 .241 \$
\end{aligned}
$$

\footnotetext{
1 “14-INV-122: La rentabilidad de los rubros agrícolas conectados a mercados internacionales en los dos estratos de la agricultura paraguaya: empresarial y familiar"
} 


\subsubsection{Acopio y Exportación}

El importe deducido a la cotización de la soja en Chicago para entrega en Mayo, denominado premio tiene su justificación que es para cubrir costo de acopio y almacenamiento. Al aplicar la fórmula de valor agregado a este eslabón, el valor agregado fue de aproximadamente 3.546 millones de dólares

Valor agregado $(\mathrm{VA})=$ Valor total de ventas - bienes intermedios

$\mathrm{VA}=($ Producción $\mathrm{x}$ precio de venta bruto $)-($ Producción $\mathrm{x}$ premio $)$

$$
\begin{aligned}
& \mathrm{VA}=\left(11.045 .971 \$ \mathrm{t} \times 371 \frac{\$}{\mathrm{t}}\right)-\left(11.045 .971 \text { ha } \times 50 \frac{\$}{\mathrm{t}}\right) \\
& \mathrm{VA}=4.098 .055 .241 \$-552.298 .550 \$ \\
& \mathrm{VA}=3.545 .756 .691 \$
\end{aligned}
$$

\subsubsection{Industrialización}

Para calcular el valor agregado en el proceso de industrialización se tuvo que previamente construir la información. La primera fue el valor de los productos procesados desde el grano de soja, a saber el aceite crudo desgomado, la harina, la cascarilla y los desechos. Según datos expuestos en la página web de CAPPRO, la producción industrializada de la zafra 2017/2018 de 3.670 .000 toneladas cuyo costo de compra fue de 371\$/t (CAPPRO, 2013b). Los sub-productos resultantes de esta cantidad de grano, y sus

\begin{tabular}{|c|c|c|c|c|}
\hline \multirow{2}{*}{ Producto } & \multicolumn{2}{|c|}{ Cantidad producida } & \multirow{2}{*}{$\begin{array}{c}\text { Precio promedio } \\
\text { USD }\end{array}$} & \multirow{2}{*}{$\begin{array}{c}\text { Valor total de } \\
\text { venta } \\
\text { USD }\end{array}$} \\
\hline & Toneladas & $\%$ & & \\
\hline $\begin{array}{l}\text { Aceite de soja } \\
\text { desgomado }\end{array}$ & 755 & $20,6 \%$ & 735,00 & 554.925 .000 \\
\hline Harina de soja & 2.573 .000 & $70,1 \%$ & 350,00 & 900.550 .000 \\
\hline Cascarilla & 202 & $5,5 \%$ & 120,00 & 24.240 .000 \\
\hline Otros (desperdicios) & 140 & $3,8 \%$ & 0 & - \\
\hline Totales & 3.670 .000 & $100,0 \%$ & & 1.479 .715 .000 \\
\hline
\end{tabular}
valores medios de exportación según el BCP () se detallan en la Tabla 1.

Tabla 1 - Industrialización y valor de venta. Periodo 2017

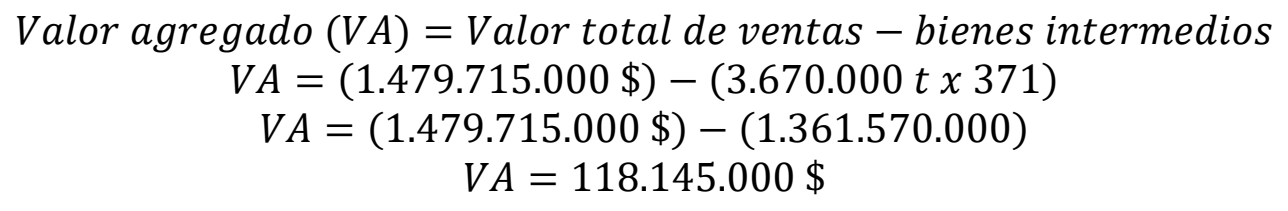

Como se puede observar en la Figura 5 en cada eslabón de la cadena aumenta el valor, el eslabón que mayor agregado aporta a la cadena es el de insumos con una ganancia de 133 \$/t (42\% del valor agregado total). En segundo lugar, se ubica la producción con 104\$/t (33\% del valor agregado total). Estos dos eslabones suman el $75 \%$ del valor agregado de la cadena. En el otro extremo están los eslabones con 
menor aporte a la agregación de valor de esta cadena. Esto son los de acopio con 43 \$/t (14\% del valor agregado total), y la industrialización con 38 \$/t o el $12 \%$ del valor agregado total.

En la Figura 1 se puede observar el precio pagado por cada eslabón de la cadena, que a su vez representa el costo de bienes intermedios para cada uno de ellos, también se puede observar el valor en porcentaje que se incrementa en cada eslabón. Cerca del 50\% del valor agregado corresponde a insumo que con la producción llegan al 75\% del valor agregado total. Básicamente los datos muestran que la cadena de la soja paraguaya tiene una elevada participación del sector primario.

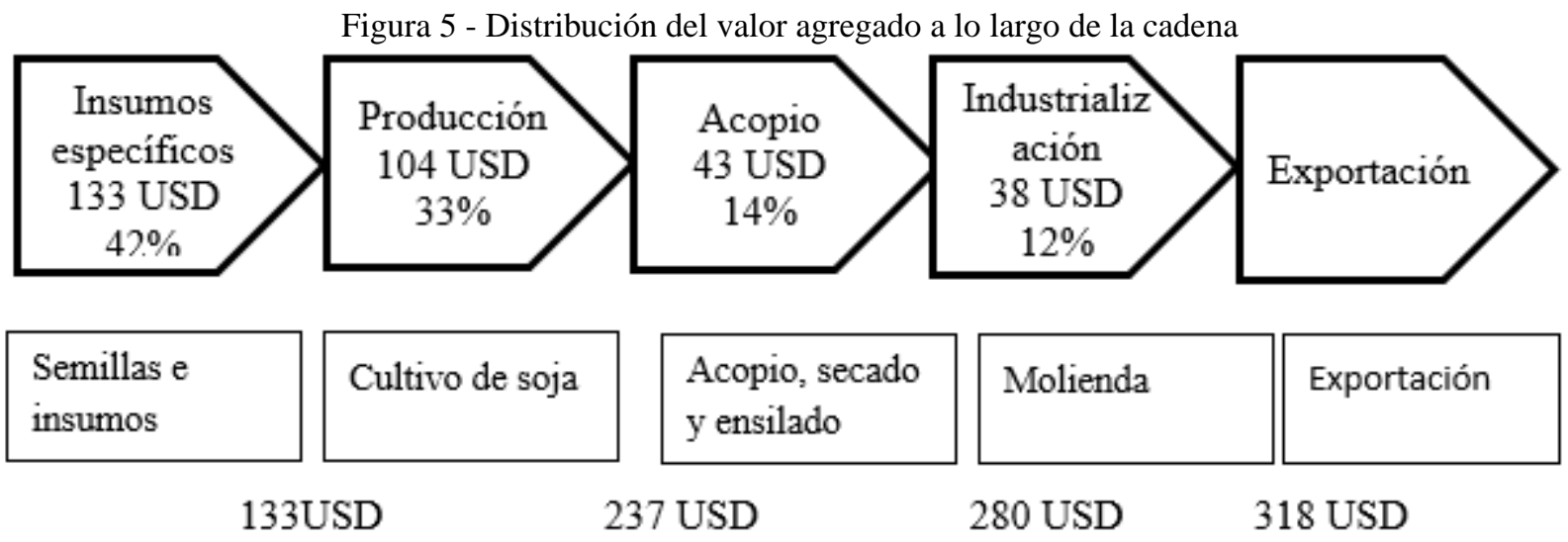

Fuente: elaboración propia

\section{CONCLUSIONES}

En relación al primer objetivo específico se han identificado los eslabones de la cadena. Con respecto al segundo objetivo específico, se ha descripto cada eslabón identificando sus actores. En lo que respecta al tercer objetivo específico, se ha analizado económicamente los eslabones de la cadena cuantificando el aporte de cada uno. La consecución de los objetivos específicos ha permitido caracteriza a la cadena concluyendo que el 50\% del valor se agregado corresponde al eslabón de producción y que el que menor valor agregado es del eslabón de industrialización. El trabajo además mostró que la industrialización de la soja en Paraguay se mantenido constante y con tendencia decreciente muy por debajo de la capacidad de molienda disponible. 


\section{REFERENCIAS}

ADUANAS.GOV. Marco filosófico. Asunción, c2010. Disponible en: https://www.aduana.gov.py/3396-1marco-filosofico.html. Acceso en 20 mar. 2019.

APROSEMP.ORG. Quienes somos. Asunción, c2017a. Disponible en: http://www.aprosemp.org.py/quienes_somos. Acceso en 20 mar. 2019.

APROSEMP.ORG. APROSEMP en números. Asunción, c2017b. Disponible en: http://www.aprosemp.org.py/uploads/capsemillera.pdf. Acceso en 20 mar. 2019.

BANCO CENTRAL DEL PARAGUAY. Informe de política monetaria-Diciembre 2015. Asunción. 2015. Disponible en: https://www.bcp.gov.py/userfiles/files/IPoM_Diciembre_2015.1\%281\%29.pdf. Acceso en: 20 mar. 2019.

BANCO CENTRAL DEL PARAGUAY. Informe de Comercio Exterior. Asunción, 2018 Disponible en: https://apps.bcp.gov.py:8444/. Acceso en 20 mar. 2019.

BANCO MUNDIAL (2014). Paraguay: Estrategia de alianza con la República del Paraguay para los años fiscales 2015-18. 2019. Washington: Disponible en: http://documents.worldbank.org/curated/en/953251468333540614/Estrategia-de-alianza-con-la-Republicadel-Paraguay-para-los-anos-fiscales-2015-18. Acceso en: 20 mar. 2019.

CADAM.ORG. Estadísticas. Asunción, cs/d. Disponible en: https://www.cadam.com.py/statistics. Acceso el 20 mar. 2019.

CAMPO, L. Apuntes de la historia económica del Paraguay. Asunción: Editora Continental. 2010, 487 p.

CAPECO.ORG. Área de siembra, producción y rendimiento. Asunción, cs/da. Disponible en: http://capeco.org.py/area-de-siembra-produccion-y-rendimiento/. Acceso el 20 mar. 2019.

CAPECO.ORG. Capacidad estática de los silos. Asunción, cs/db. Disponible en: http://capeco.org.py/capacidad-estatica-de-silos-es/. Acceso el 20 mar. 2019.

CAPECO.ORG. Molienda de granos. Asunción, cs/dc. Disponible en: http://capeco.org.py/soja-es-mol-gran/. Acceso el 20 mar. 2019.

CAPPRO. Quienes somos. c2013a. Disponible en: http://cappro.org.py/la-camara-2. Acceso el 20 mar. 2019

CAPPRO. Estadísticas. c2013b. Disponible en: http://cappro.org.py/estadisticas. Acceso el 20 mar. 2019

CAPPRO. Boletín estadístico diciembre 2017. Asunción, 2018 4p. Disponible en: http://cappro.org.py/wpcontent/uploads/2013/11/Bolet\%C3\%ADn-CAPPRO_Diciembre_2017.pdf. Acceso el 20 mar. 2019

CINDA.ORG. Quienes somos. Asunción, c2019. Disponible en: http://www.cinda.org.py/asociados.php. Acceso el 20 mar. 2019.

CHÁVEZ, J. Cadena de valor, estrategias genéricas y competitividad: el caso de los productores de café orgánico del Municipio de Tanetze de Zaragoza, Oaxaca. (2012). Disponible en: http://www.eumed.net/libros-gratis/2013b/1345/1345.pdf. Acceso en: 20 mar. 2019.

FAO. Desarrollo de cadenas de valor alimentarias sostenibles: Principios rectores. 2015. Disponible en: http://www.fao.org/3/a-i3953s.pdf. Acceso en: 20 mar. 2019. 
FONDO MONETARIO INTERNACIONAL (FMI). Primary Commodity Prices. Washington, 2018. Disponible en: http://www.imf.org/external/np/res/commod/External_Data.xls. Acceso en: 20 mar. 2019.

HEREDIA, J.; HUARACHI J. (2009). El índice de la ventaja comparativa revelada (VCR) entre el Perú y los principales exportadores del mundo: El caso de la Región Lambayeque. Cuadernos de Difusión v.

14, n. 26, p. 27-55, jun. 2009 Disponible en: https://www.esan.edu.pe/publicaciones/cuadernos-dedifusion/26/Heredia-Huarachi.pdf. Acceso en: 20 mar. 2019.

IPTA.GOV. Marco Legal. Asunción, cs/d. Disponible en: http://www.ipta.gov.py/index.php/marco-legal. Acceso el 20 mar. 2019.

MINISTERIO DE AGRICULTURA Y GANADERÍA. Síntesis estadísticas producción agropecuaria año agrícola 2017/2018. San Lorenzo. 2018, 50 p. Disponible en: http://www.mag.gov.py/Censo/SINTESIS\%20Estadisticas\%202017_2018\%20_pdf\%20NOV.pdf. Acceso en: 20 mar. 2019.

MTESS.GOV. Asunción, cs/d. Disponible en: http://www.mtess.gov.py/index.php/vision-y-mision. Acceso el 20 mar. 2019.

MIC.GOV. Institucional. Asunción, c2014. Disponible en: http://www.mic.gov.py/mic/site/mic/institucional.php. Acceso el 20 mar. 2019.

OFICINA ECONÓMICA Y COMERCIAL DE ESPAÑA EN ASUNCIÓN. Informe económico y comercial. Paraguay 2017. Estudios e Informes. Madrid. 2017, 42 p. Disponible en: https://www.icex.es/icex/es/navegacion-principal/todos-nuestros-servicios/informacion-demercados/paises/navegacion-principal/el-mercado/estudios-informes/DOC2016655809.html?idPais=PY. Acceso en: 20 mar. 2019.

ORTIZ, E.; RIQUELME, C. Paraguay: Potencia agroindustrial para alimentar al mundo. Es hora de marcar el antes y el después... Asunción: AGR. 2016,144 p. Disponible en: http://cappro.org.py/wpcontent/uploads/2016/09/Libro-Agroindustria_Agosto-2016.pdf. Acceso en: 20 mar. 2019.

PORTER M. Ventaja competitiva. Quinta reimpresión. México: CECSA. 2006, 550 p.

SENAVE.GOV. Quienes somos. Asunción, cs/d. Disponible en: http://www.senave.gov.py/quienessomos.html. Acceso el 20 mar. 2019.

SET.GOV. Institucional. Asunción, cs/d. Disponible en: https://www.set.gov.py/portal/PARAGUAYSET/Documentos/Institucional. Acceso el 20 mar. 2019.

SETRINI, G. Cadenas de valor y pequeña producción agrícola en el Paraguay. Asunción, 2014, 60 p. Disponible en: http://biblioteca.clacso.edu.ar/Paraguay/cadep/20160713045421/6.pdf. Acceso en: 20 mar. 2019.

SPRINGER-HEINZE, A. (2008). ValueLinks Manual: The Methodology of Value Chain Promotion. Eschborn. 2008, 243 p. Disponible en: http://www2.giz.de/wbf/4tDx9kw63gma/ValueLinks_Manual.pdf. .Acceso en: 20 mar. 2019. 